\title{
Macular Morphology in Patients with Retinopathy of Prematurity
}

\author{
Silviya Krumova ${ }^{*}{ }^{(0)}$, Desislava Voynikova ${ }^{2}$, Desislava Koleva-Georgieva ${ }^{1}$, \\ Vasil Marinov' ${ }^{1}$, Nelly Sivkova ${ }^{1}$ \\ ${ }^{1}$ Department of Ophthalmology, Faculty of Medicine, Medical University of Plovdiv, Plovdiv, Bulgaria \\ ${ }^{2}$ University of Plovdiv "Paisii Hilendarski”, Plovdiv, Bulgaria \\ Email: *krumova_silviya@abv.bg
}

How to cite this paper: Krumova, S., Voynikova, D., Koleva-Georgieva, D., Marinov, V. and Sivkova, N. (2020) Macular Morphology in Patients with Retinopathy of Prematurity. Open Journal of Ophthalmology, 10, 59-68.

https://doi.org/10.4236/ojoph.2020.101008

Received: November 14, 2019

Accepted: January 5, 2020

Published: January 8, 2020

Copyright ( 2020 by author(s) and Scientific Research Publishing Inc. This work is licensed under the Creative Commons Attribution International License (CC BY 4.0).

http://creativecommons.org/licenses/by/4.0/

(c) (i) Open Access

\begin{abstract}
Purpose: To investigate macular morphology by spectral domain optical coherence tomography (SDOCT) in children with history of the retinopathy of prematurity (ROP). Materials and methods: In this cross-sectional study we included 35 eyes of 18 patients, aged 4 to 8 years, with history of ROP. Twenty-one eyes had received treatment for type 1 pre-threshold ROP. The others showed spontaneous regression of the disease. A $7 \times 7-\mathrm{mm}$ retinal area was sampled using a 3-dimensional scanning protocol with high-resolution spectral domain optical coherence tomography (SOCT Copernicus REVO). The central foveal thickness, mean macular thickness, macular volume and foveal depression were measured. The macular anatomy and the foveal contour were examined. Results: The mean birth weight was $1338.29 \pm 324.05$ grams. Mean gestational age at birth was $29.37 \pm 2.07$ weeks. Twenty-one eyes were treated with laser or cryotherapy. All of them showed regression of the disease. The mean best corrected visual acuity was $20 / 28$. The average central foveal thickness was $262.26 \pm 37.39$, with $68.57 \%$ of preterm $>240 \mu \mathrm{m}$ and $22.86 \%>$ $220 \mu \mathrm{m}$. In all eyes, the foveal contour is abnormal. A foveal depression was absent in 25 eyes (71\%). Conclusion: In the majority of children with a history of ROP, we found changes in macular morphology, including retention of inner retinal layers and absent foveal depression. In our study foveal hypoplasia was associated with good functional outcome.
\end{abstract}

\section{Keywords}

Foveal Pit, Macular Morphology, Optical Coherence Tomography, Preterm, Visual Acuity

\section{Introduction}

Retinopathy of the prematurity (ROP) remains among the leading causes of ir- 
reversible but preventable childhood blindness worldwide.

Retinopathy of the prematurity belongs to the group of socially significant diseases due to the fact that the lack of adequate and timely detection and treatment of the disease leads to permanent blindness, disability of the individual and socio-economic consequences. More than 50,000 children worldwide have been blinded by ROP. Many more are expected to be unilaterally blind or with reduced visual acuity. Due to the development of neonatal care, the survival rate of premature babies has increased in recent years. Therefore, understanding the anatomy of these eyes and causes for visual impairment will be crucial for ophthalmologist [1].

Optical coherence tomography (OCT) is a noninvasive, noncontact method that uses low-coherence interferometry to perform high-resolution cross-sectional imaging of tissue morphologic features, providing an "optical biopsy" [2]. Optical coherence tomography enables evaluation of macular anatomy and detection of causes of decreased visual acuity. Few studies have evaluated ROP maculopathy and some are limited by the use of traditional time-domain OCT. Spectral domain optical coherence tomography (SDOCT) provides higher resolution (up to $5 \mu \mathrm{m}$ ) and decreased acquisition time in comparison to the previous time-domain OCT. This is extremely useful while performing OCT examination in uncooperative children. Disturbances in the normal development of fovea in patients with a history of ROP lead to morphological abnormalities, seen by SDOCT. Many factors affect foveal development: prematurity, development of vasculature, foveal tissue elasticity, intraocular pressure, and retinal stretching [3] [4]. Some of them act synergistically. According to Mietz et al. (1992) a more appropriate term is foveal hypoplasia from aplasia because hypoplasia involves both partial and complete lack of structure [5]. Recent studies have shown that SDOCT can be used as a diagnostic aid and prognostic indicator for the foveal hypoplasia [6].

The present study was performed to determine the SDOCT findings in patients with history of ROP.

\section{Methods}

We evaluated 35 eyes of 18 children with history of any signs of ROP. Twenty-one eyes received treatment for type 1 prethreshold ROP. The others showed spontaneous regression of the disease. Fifteen patients reached stage 3 of prethreshold ROP type 1 with "plus disease" in zone 2, and 6 patients, stage 2 with "plus disease" in zone 2. Sixteen eyes were treated with cryotherapy and 5 eyes were treated with laser therapy. All of them showed regression of the disease. This study was performed in accordance with the Declaration of Helsinki and was approved by the Local Ethics Committee of the participating center. Written informed consent was obtained from both parents of the children after explaining the imaging modality to them and to the child. Inclusion criteria were type 1 and 2 prethreshold ROP, as well as AP-ROP. Patients with stage 4 and 5 ROP were not 
included. Autorefractometry, best corrected visual acuity, slit-lamp biomicroscopy, dilated pupil indirect fundus examination, and intraocular pressure measurements (using a noncontact tonometer) were performed. Pupils were dilated with three drops of cyclopentolate $1 \%$ at intervals of fifteen minutes, and the cycloplegic autorefraction (UNICOS URK-700 autokeratorefractometer) was assessed 30 - 35 minutes after the last drop was instilled. The measurements performed by autokeratorefractometer were repeated at least 5 times, and the average value was recorded for each eye. In addition, the spherical equivalent refraction values $(\mathrm{SE}=$ spherical error $+50 \%$ of cylindrical error) was calculated from the average refractive error values for each eye. Data regarding gestational age at birth, sex, and history of types of treatment were recorded. Patients were examined once.

\subsection{Optical Coherence Tomography Measurements}

Images of the macular region were obtained using a SOCT Copernicus REVO (Optopol Technology SA, Zawiercie, Poland). This instrument uses a super luminescent diode light source with a peak wavelength of $830 \mathrm{~nm}$ (bandwidth, 50 $\mathrm{nm}$ ), providing OCT images with an axial resolution of $5 \mu \mathrm{m}$, a transverse resolution of $12 \mu \mathrm{m}$ to $18 \mu \mathrm{m}$, and a scanning speed of 80.000 A-scans per seconds. All included patients underwent SDOCT examination by a single operator. Internal fixation was used to suppress ocular movements. Multiple measurements were taken and the best centered one with good signal strength was chosen for analysis. The programs used for the present study were 3D scan protocols. The macular thickness data are presented in the nine ETDRS areas (Early Treatment Diabetic Retinopathy Study Research Group, 1985). The central subfield (A1) measures $1 \mathrm{~mm}$ in diameter, the inner (A2 - A5) and outer (A6 - A9), 3 and $6 \mathrm{~mm}$, respectively [7]. The central foveal thickness as well as nasal and temporal parafoveal thickness were measured. The mean of parafoveal thickness was calculated. The foveal depression was calculated by subtracting the central foveal thickness from the mean parafoveal thickness. Values below $56 \mu \mathrm{m}$ were taken as an indication of lack of foveal depression [8].

The patients were divided into two groups. The first group included patients with ROP who underwent laser or cryotherapy (21 eyes). The second group included patients with retinopathy of prematurity, in which spontaneous regression of the disease occurred (14 eyes).

\subsection{Statistical Analysis}

The data of 35 eyes of the 18 subjects were analysed using SPSS 19.0. Descriptive statistics were reported as mean and standard deviation. The normality of the distribution of the study sample was assessed by the Kolmogorov-Smirnov test. For statistical processing of the data from both groups we used the Independent two-sample t-test. In the prematurely born children, forward linear regression analyses were carried out to evaluate how much the variation in the central fo- 
veal thickness and foveal depression could be explained by gestational age, birth weight, visual acuity and spherical equivalent. $\mathrm{P}$ values less than 0.05 were considered to be statistically significant.

\section{Results}

Thirty-five eyes of 18 patients were examined. The mean age of the children was $6.06 \pm 1.11$ years. The mean gestational age was $29.37 \pm 2.07$ weeks and the mean birth weight was $1338.29 \pm 324.05$ grams. The average visual acuity was 20/28 and mean SE was $-1.46 \pm 3.05$ diopters. On OCT analysis, the mean central foveal thickness was $262.26 \pm 37.39 \mu \mathrm{m}$, mean parafoveal thickness was $292.00 \pm$ $23.52 \mu \mathrm{m}$, mean macular volume was $7.83 \pm 0.37 \mu \mathrm{m}$, and mean foveal depression was $29.74 \pm 33.28 \mu \mathrm{m}$. A foveal depression was absent in 25 eyes (71\%).

The spherical equivalent was highly correlated to visual acuity (Figure 1). We found that the absence of foveal depression correlated significantly with gestational age and birth weight (Figure 2, Figure 3).

Group 1 consisted of 21 eyes of 11 children (60\%), with a mean gestational age was $28.48 \pm 1.5$ weeks, mean birth weight was $1173.33 \pm 170.07$ grams, mean visual acuity was $20 / 30$, and spherical equivalent was $-2.33 \pm 2.45 \mathrm{D}$. The mean central foveal thickness was $277.76 \pm 34.0 \mu \mathrm{m}$, mean macular thickness was

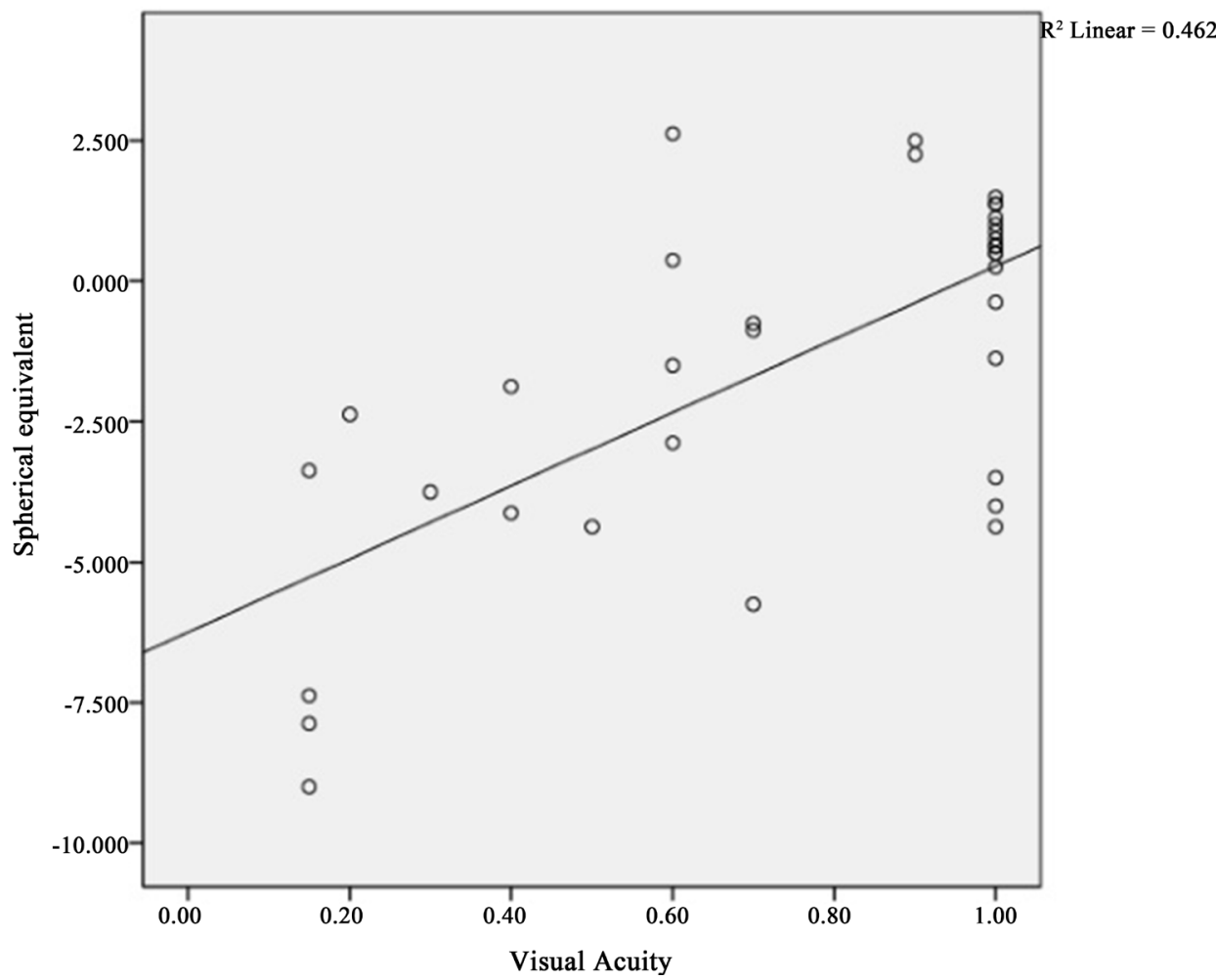

Figure 1. The plot of the visual acuity and spherical equivalent in children with ROP indicates that patients with higher myopia have lower visual acuity $(p<0.0001)$. Linear regression analysis was used to find a significant correlation between visual acuity (horizontal axis) and spherical equivalent (vertical axis). 


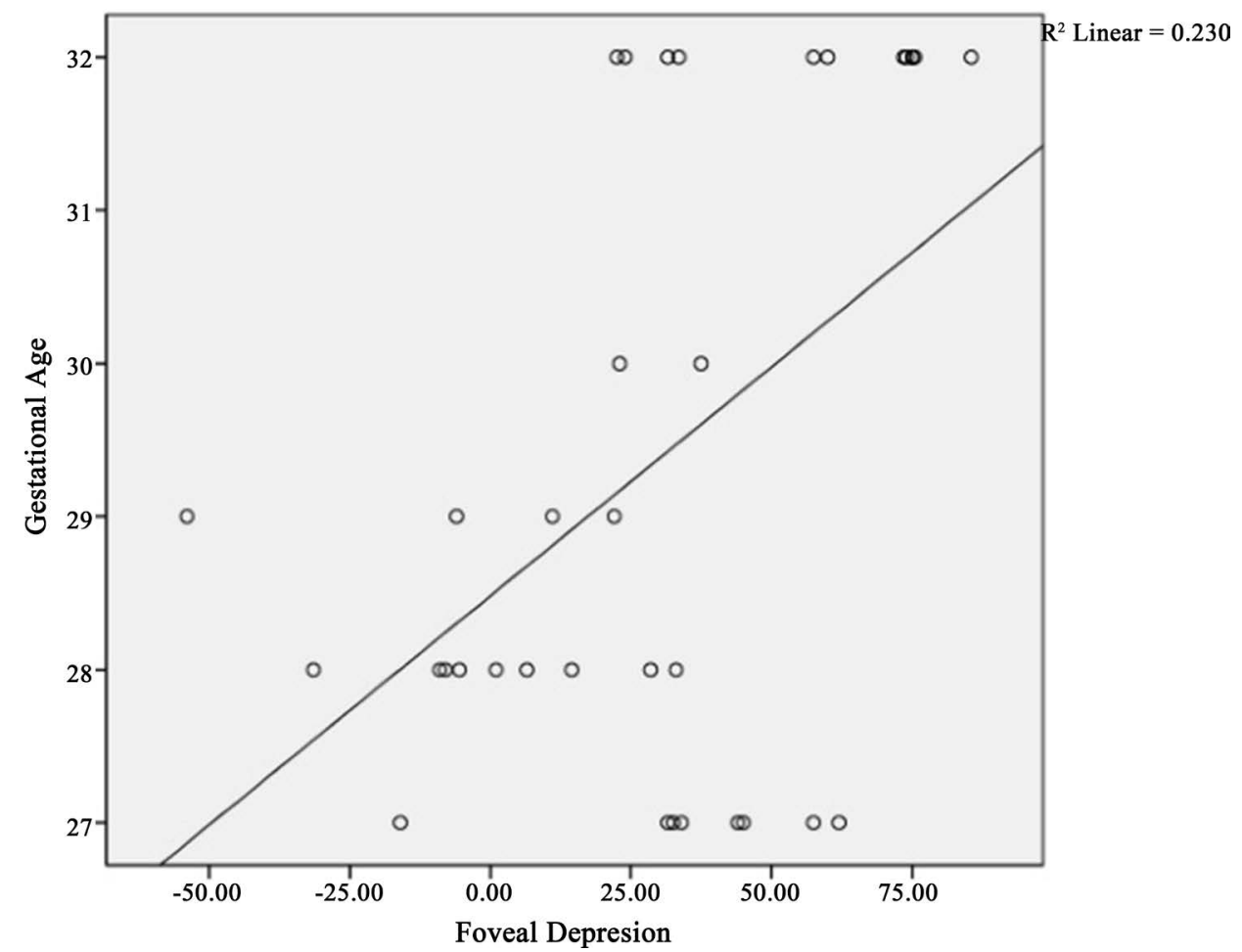

Figure 2. The plot of gestational age (vertical axis) in weeks and foveal depression in children with ROP (horizontal axis) shows a significant association between later gestational age and improved foveal depression $(p=0.004)$. Linear regression analysis was used.

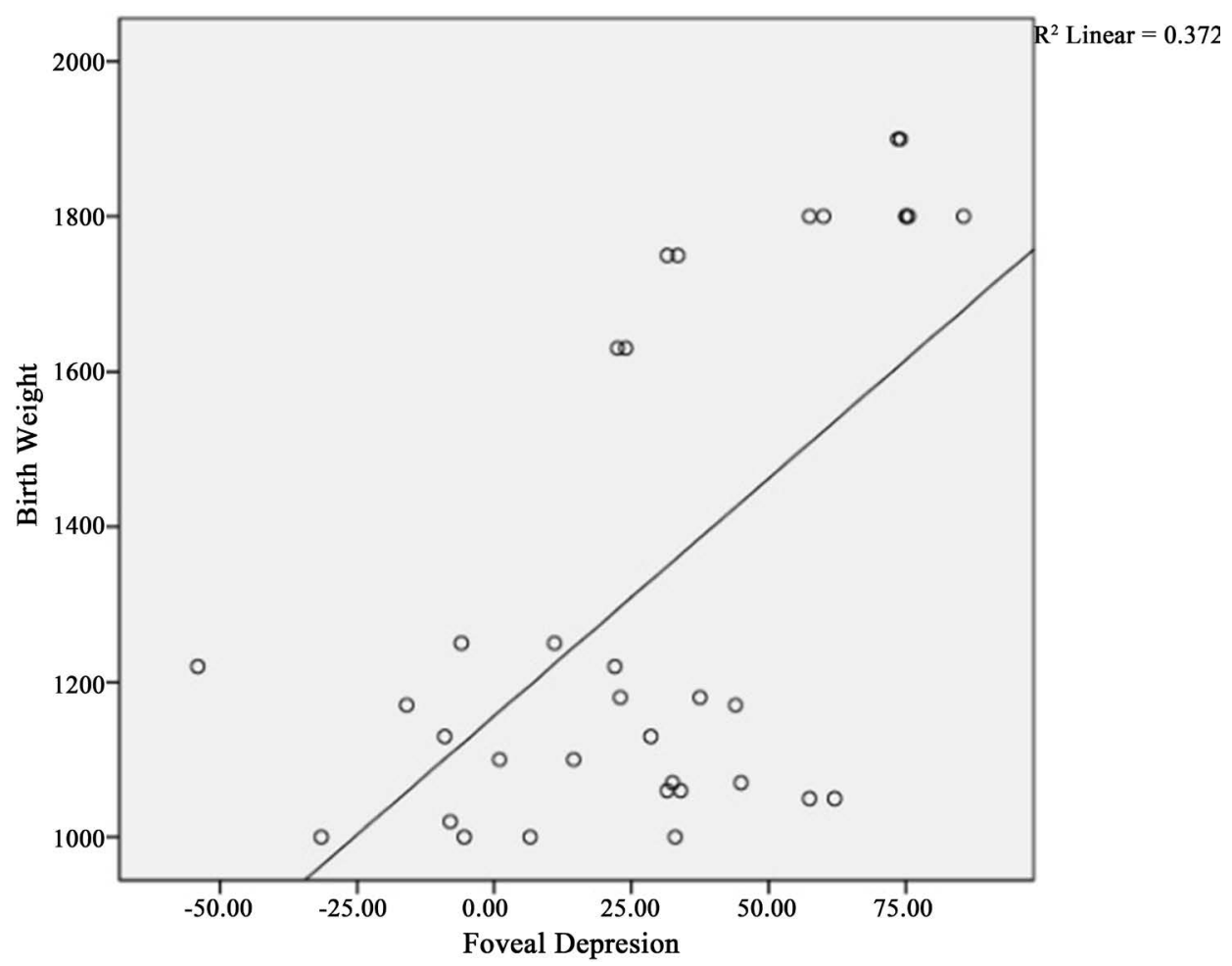

Figure 3. The plot of birth weight (vertical axis) in grams and foveal depression in children with ROP (horizontal axis) shows a significant association between higher birth weight and foveal depression $(p<0.0001)$. Linear regression analysis was used. 
$276.62 \pm 25.03 \mu \mathrm{m}$, mean parafoveal thickness was $291.05 \pm 26.04 \mu \mathrm{m}$, mean macular volume was $7.88 \pm 0.34$ and mean foveal depression was $13.29 \pm 24.01$ $\mu \mathrm{m}$. A foveal depression was absent in 21 eyes (100\%).

Group 2 consisted of 14 eyes of 7 children (40\%), with a mean gestational age of $30.71 \pm 2.13$ weeks, mean birth weight $1583.71 \pm 370.78$ grams, mean visual acuity was $20 / 25$, and spherical equivalent $-0.16 \pm 3.47 \mathrm{D}$. The mean central foveal thickness was $239.00 \pm 30.12 \mu \mathrm{m}$, mean macular thickness was $283.27 \pm$ $15.35 \mu \mathrm{m}$, mean parafoveal thickness was $293.43 \pm 19.99 \mu \mathrm{m}$, mean macular volume was $7.79 \pm 0.39$ and mean foveal depression was $54.43 \pm 30.28 \mu \mathrm{m}$. A foveal depression was absent in 4 eyes $(28.6 \%)$.

In this study we found statistically significant difference in the central foveal thickness between children treated for ROP and those with spontaneous regression $(p=0.002)$. There was no significant difference in mean parafoveal thickness, mean macular thickness, and the macular volume between the two groups ( $p=0.774, p=0.382, p=0.502$ respectively). The absence of foveal depression was observed significantly more often in children that underwent treatment for ROP $(p>0.001)$. Children with spontaneous regression showed significantly higher visual acuity $(p=0.047)$. Myopia was significantly more common in children from the first group $(p=0.037)$.

\section{Discussion}

In this cross-sectional study, we found that patients with ROP had macular abnormalities seen with SDOCT. We found that significant number of patients with history of ROP had absence of foveal depression and retention of the layer of retinal ganglion cells, inner plexiform layer, and inner nuclear layer at the foveal center (Figure 4). These findings are consistent with similar studies that have been performed in Asian patients by $\mathrm{Wu}$ et al. [8].

Morphologically, the adult fovea is marked by a pit that is dense with photoreceptors and devoid of overlying inner retinal layers and vasculature. The initial stage of foveal development begins in the $22^{\text {nd }}$ week of gestational age. Formation of foveal depression starts in the $24^{\text {th }}$ to $28^{\text {th }}$ week of gestational age and the excavation is complete 15 to 45 months after birth [9] [10]. The process of foveal maturation has been described as a centrifugal displacement of cells of the inner retina toward the periphery, centripetal migration of cone photoreceptors toward the location of the incipient fovea, and cone specialization of the foveolar cones [10]. Foveal depression continues to deepen up to 15 months after birth due to centrifugal displacement of retinal internal cells. This is considered as a complete extrusion from the inner nuclear and plexiform layers behind the foveola. The centripetal migration of the cone photoreceptors is represented by the outer nuclear layer widening. Cone specialization is characterized by a decrease in diameter and an increase in the length of the outer segment of the cone. This allows an increase in foveolar cone packing density. The cone specialization is represented on OCT by the outer segment elongation [11]. Photoreceptor 


\begin{tabular}{ll|l|l|} 
ID: 436 & Comments: & \\
DOB: $27 / 05 / 2010$ & Gender: & Male & \\
Age: 8 & Eye: & & \\
\hline
\end{tabular}

L 19/12/2018 13:55:35

RETINA | TOMOGRAM
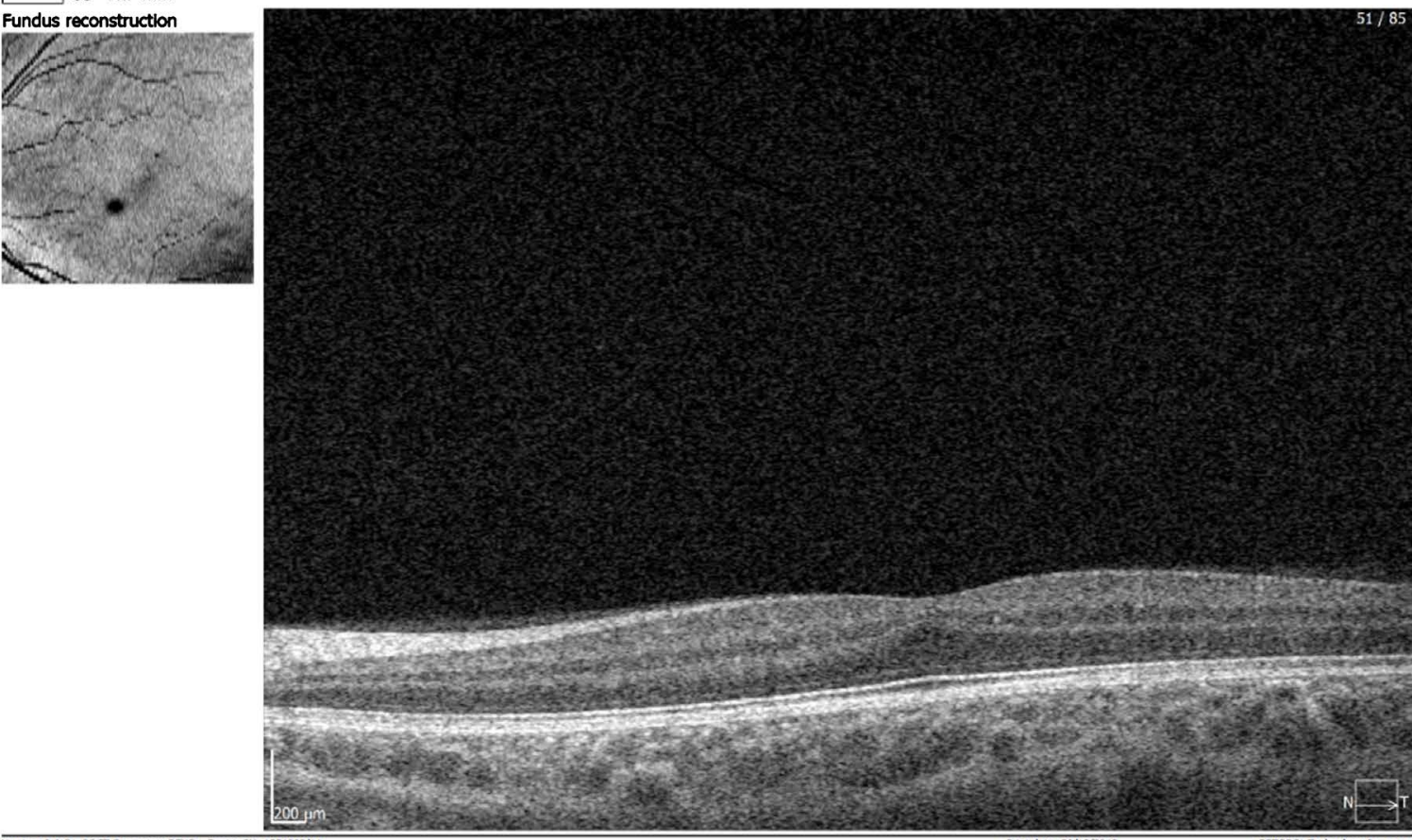

\begin{tabular}{|c|c|c|c|c|c|}
\hline \multicolumn{5}{|c|}{ Version: 8.0 .2 SOCT Coperniaus REVO Device SN: 1551062/11 } & CoTOPQ Tedriology SD. 20.0. \\
\hline & & & & Comments: & \\
\hline & 437 & Exam date & $19 / 12 / 2018$ & & \\
\hline DOB: & $13 / 05 / 2012$ & Gender: & Male & & \\
\hline Age: & 6 & Eye: & Right & & \\
\hline
\end{tabular}

\begin{tabular}{|l|l|l|l|l}
\hline Age: & 6 \\
\hline & 3D $7 \times 7 \mathrm{~mm}$
\end{tabular}

Eye: Right

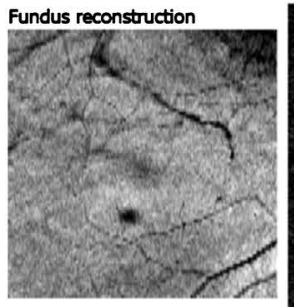

RETINA | TOMOGRAM

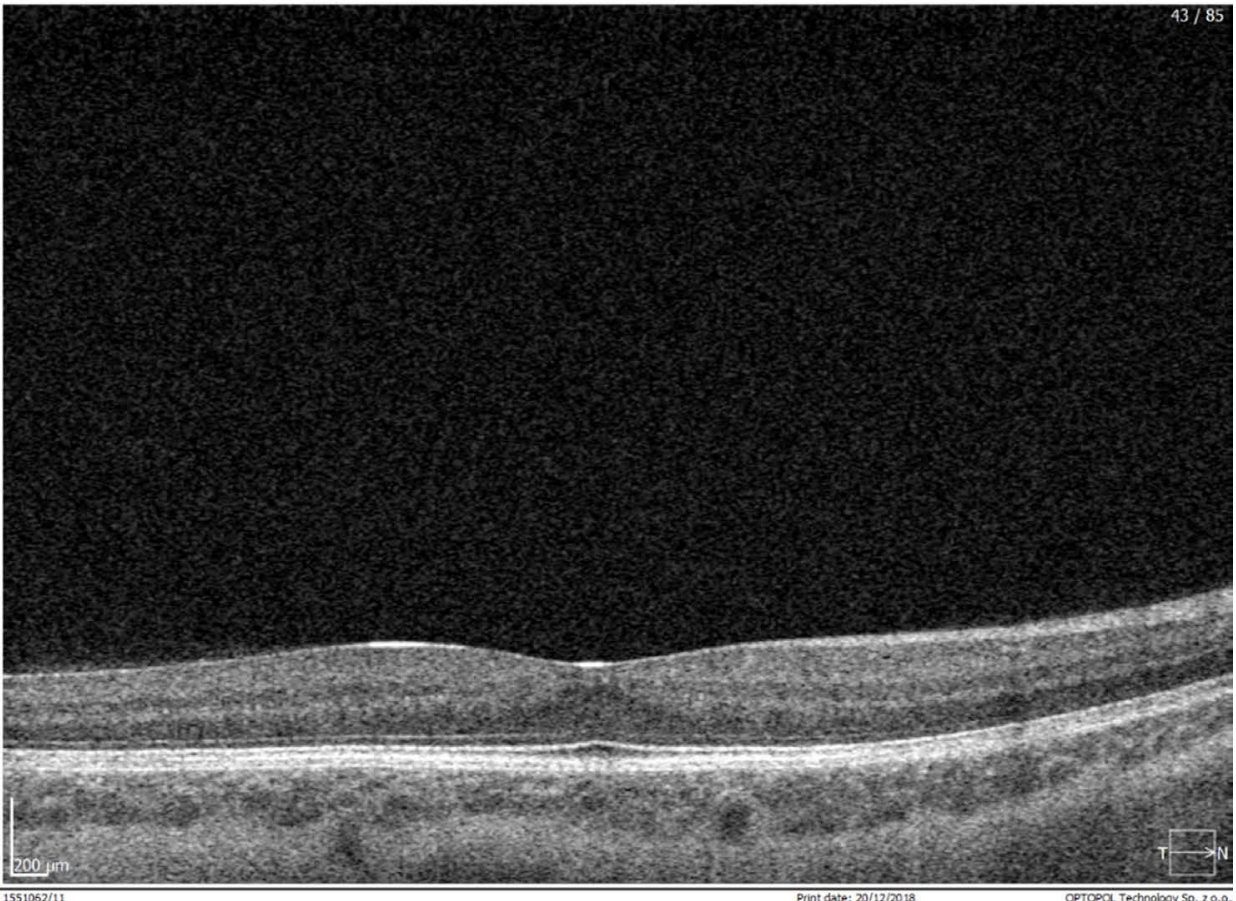

Figure 4. Spectral domain optical coherence tomography images demonstrating flattened foveal contour in two eyes with retinopathy of prematurity and visual acuity of 20/20 (Top), and 20/25 (Bottom). Cryotherapy was performed on both patients. 
differentiation with outer segment elongation occurs almost entirely after birth and continues until 4 years of age [7] [12]. Maldonado and al. found that prematurely born children have a thin photoreceptor layer seen on SDOCT [12]. There are studies that do not report photoreceptor abnormalities in patients with a history of ROP [8], which is consistent with the data from the Villegas and al. [13], which found maturation by outer segment elongation in $96 \%$ of the eyes. This is supported by other studies that demonstrate that significant foveal cone specialization may occur in the absence of a foveal pit [14]. Inner retinal layer migration appears to be independent from photoreceptor development [11] [15]. Our study found that $71 \%$ of patients had no foveal depression, with an average visual acuity of $20 / 30$ in these children. This result is confirmed by Villegas and al. who found that $91 \%$ of patients with visual acuity of $20 / 40$ or better did not have foveal depression [13]. This is confirmed by Marmor et al. found that the specialization of foveal cone can be preserved both anatomically and functionally, despite the absence of a foveal pit. They described adults with good visual acuity and absent foveal reflex on OCT. These subjects had cones of normal diameter. To describe this condition, Marmor et al. introduced the term fovea plana [11].

Our study found that children treated for ROP had higher central foveal thickness than children who develop spontaneous regression of the disease. Accordingly, the lack of a foveal pit was significantly higher in the treated children.

The average visual acuity for both groups was $20 / 30$, but significantly higher in premature children was in the second group. In this study we found significant correlation between visual acuity and refractive error. Myopic patients were more likely to have decreased best corrected visual acuity.

$\mathrm{Wu}$ and al. found that low gestational age significantly correlated with greater central foveal thickness [8]. Unlike them we found no such correlation ( $p=$ $0.87)$.

The limitations of our study include only patients able to cooperate with OCT scanning were enrolled, thus introducing a patient selection bias. This potentially correlates with the fact that no patients had a visual acuity less than or equal to $20 / 200$, as these patients were unlikely to fixate on a scanning target. Also, our study is limited by a small sample size.

\section{Conclusion}

In summary, SDOCT revealed high percentage of macular abnormalities in children with history of retinopathy of prematurity, including retention of inner retinal layers and absent foveal depression. We consider macular abnormalities as a useful warning sign for patients with a history of ROP. This is not always associated with reduced visual acuity. In our study foveal hypoplasia was associated with good functional outcome. We found, using the SDOCT that prematurely born children treated for ROP have thicker central fovea than children who showed spontaneous regression. This may be due to an arrest in 
the lateral migration of cells during normal development of the fovea.

\section{Conflicts of Interest}

The authors declare no conflicts of interest regarding the publication of this paper.

\section{References}

[1] Cryotherapy for Retinopathy of Prematurity Cooperative Group (2006) Visual Acuity at 10 Years in Cryotherapy for Retinopathy of Prematurity (CRYO-ROP) Study Eyes: Effect of Retinal Residua of Retinopathy of Prematurity. Archives of Ophthalmology, 124, 199-202. https://doi.org/10.1001/archopht.124.2.199

[2] Huang, D., Swanson, E.A., Lin, C.P., et al. (1991) Optical Coherence Tomography. Science, 254, 1178-1781. https://doi.org/10.1126/science.1957169

[3] Springer, A.D. and Hendrickson, A.E. (2004) Development of the Primate Area of High Acuity. Use of Finite Element Analysis Models to Identify Mechanical Variables Affecting Pit Formation. Visual Neuroscience, 21, 53-62. https://doi.org/10.1017/S0952523804041057

[4] Ecsedy, M., Szamosi, A., Karko, C., et al. (2007) A Comparison of Macular Structure Imaged by Optical Coherence Tomography in Preterm and Full-Term Children. Investigative Ophthalmology \& Visual Science, 48, 5207-5211. https://doi.org/10.1167/iovs.06-1199

[5] Mietz, H., Green, W.R., Wolff, S.M., et al. (1992) Foveal Hypoplasia in Complete Oculocutaneous Albinism: A Histopathologic Study. Retina, 12, 254-260.

https://doi.org/10.1097/00006982-199212030-00011

[6] Holmstrom, G., Eriksson, U., Hellgren, K., et al. (2010) Optical Coherence Tomography Is Helpful in the Diagnosis of Foveal Hypoplasia. Acta Ophthalmologica, 88, 439-442. https://doi.org/10.1111/j.1755-3768.2009.01533.x

[7] Early Treatment Diabetic Retinopathy Study Research Group (1985) Photocoagulation for Diabetic Macular Edema. Early Treatment Diabetic Retinopathy Study Report Number 1. Archives of Ophthalmology, 103, 1796-1806. https://doi.org/10.1001/archopht.1985.01050120030015

[8] Wu, W.C., Lin, R.I., Shih, C.P., et al. (2012) Visual Acuity, Optical Components, and Macular Abnormalities in Patients with a History of Retinopathy of Prematurity. Ophthalmology, 119, 1907-1916. https://doi.org/10.1016/j.ophtha.2012.02.040

[9] Hendrickson, A.E. and Yuodelis, C. (1984) The Morphological Development of the Human Fovea. Ophthalmology, 91, 603-612. https://doi.org/10.1016/S0161-6420(84)34247-6

[10] Yuodelis, C. and Hendrickson, A. (1986) A Qualitative and Quantitative Analysis of the Human Fovea during Development. Vision Research, 26, 847-855. https://doi.org/10.1016/0042-6989(86)90143-4

[11] Marmor, M.F., Choi, S.S., Zawadzki, R.J., et al. (2008) Visual Insignificance of the Foveal Pit: Reassessment of Foveal Hypoplasia as Fovea Plana. Archives of Ophthalmology, 126, 907-913. https://doi.org/10.1001/archopht.126.7.907

[12] Maldonado, R.S., O'Connell, R.V., Sarin, N., et al. (2011) Dynamics of Human Foveal Development after Premature Birth. Ophthalmology, 118, 2315-2325. https://doi.org/10.1016/j.ophtha.2011.05.028

[13] Villegas, V.M., Capo, H., Cavuoto, K.M., et al. (2014) Foveal Structure-Function Correlation in Children with History of Retinopathy of Prematurity. American 
Journal of Ophthalmology, 158, 508-512. https://doi.org/10.1016/j.ajo.2014.05.017

[14] McAllister, J.T., Dubis, A.M., Tait, D.M., et al. (2010) Arrested Development: High-Resolution Imaging of Foveal Morphology in Albinism. Vision Research, 50, 810-817. https://doi.org/10.1016/j.visres.2010.02.003

[15] Thomas, M.G., Kumar, A., Mohammad, S., et al. (2011) Structural Grading of Foveal Hypoplasia Using Spectral-Domain Optical Coherence Tomography a Predictor of Visual Acuity? Ophthalmology, 118, 1653-1660.

https://doi.org/10.1016/j.ophtha.2011.01.028 